\title{
Constitutive Expression of P67 Increases cPKCalpha Level in C2C12 Myoblasts and Decreases in Myotubes
}

\author{
Samit K. Datta, Bansidhar Datta* \\ Department of Chemistry and Biochemistry, Kent State University, Kent \\ bdatta@kent.edu
}

\begin{abstract}
During skeletal muscle differentiation several growth factor mediated signaling pathways must shut off to allow cycling myoblasts to permanently withdraw from cell cycle and fuse into multinucleated myotubes. The level of eukaryotic initiation factor 2 (eIF2)-associated glycoprotein p67 gradually increases when C2C12 myoblasts are differentiating into myotubes. At the same time, the level of lipid induced expression and activity of protein kinase C (cPKCalpha) gradually decreases. cPKCalpha is activated in response to growth factors and sends signal to downstream effectors for cell growth and proliferation and differentiation. We therefore examined whether constitutive expression of rat p67 has any effect on expression of PKC both in C2C12 myoblasts and myotubes. We found 10 -fold increase in cPKCalpha level in p67-overexpressing myoblasts and p67's N-terminal lysine-rich domains I \& II and in some extend its acidic residue-rich domain are essential for this activity. Although, p67's conserved D251 residue alone has no effect of cPKCalpha level, this residue is however needed to cooperate with the function(s) of lysine-rich domains I \& II. The later cooperation was prominent in differentiated myoblasts where cPKCalpha level was down in p67-overexpressing myotubes. In addition, p67's another conserved residue H331 also cooperates with the functions lysine-rich domains I \& II in lowering the level of cPKCalpha in C2C12 differentiated myotubes. Altogether, our data suggest that increased p67 level is involved in increasing the level of cPKCalpha in C2C12 myoblasts and decreasing in the myotubes.
\end{abstract}

\begin{abstract}
Abbreviations Used: p67, a $67 \mathrm{kDa}$ glycoprotein that binds to both eukaryotic initiation factor 2 (eIF2) and extracellular signal-regulated kinases 1 \& 2 (ERK1/2); D251A, a p67 point mutant, which has alanine substitution for aspartic acid at 251 amino acid residue; D6/2, a p67 block mutant, where a stretch of $N$ terminal acidic amino acid residues has been replaced with uncharged amino acids; K1K2, a p67 block mutant, where both of its $\mathrm{N}$-terminal lysine/arginine-rich stretches of amino acid residues have been replaced with uncharged amino acids; D6/2+D251A, a D6/2 mutant containing a second site point mutation, where its 251 aspartic amino acid residue has been changed to alanine; D6/2+H331A, a D6/2 mutant containing a second site point mutation, where its histidine amino acid residue at position 331 has been changed to alanine; cPKCalpha, Protein kinase $C$; and V, vector expressing enhanced green fluorescence protein (EGFP).
\end{abstract}

Keywords: Protein kinase C; C2C12 myoblasts and myotubes; differentiation; eIF2-associated protein p67

\section{INTRODUCTION}

Skeletal muscle differentiation is a highly orchestrated cellular process that includes a series of steps during the fusion of myogenic cells into myotubes (1). Among several processes, the shutting off the growth promoting signals mediated by growth factors is absolutely required for myoblasts to permanently withdraw from cell cycle (1-2). Several growth-promoting signals maintain the muscle stem cells (satellite myogenic cells) in cycling stage. For example, (i) extracellular signal-regulated kinases 1 and 2 (ERK1/2) MAP kinases (3-4), (ii) Integrin-mediated cell signaling via activation of focal adhesion kinase (FAK) (5-6), (iii) lipid induced protein kinase C (cPKCalpha) (7-8), and several others send potent growth promoting signals to the myogenic cells. $\mathrm{C} 2 \mathrm{C} 12$ mouse myoblasts are the excellent model system to study the details of myogenesis. In $\mathrm{C} 2 \mathrm{C} 12$ myoblasts, the expression and activity of cPKCalpha is very high and this decreases during the course of differentiation of myoblasts into myotubes and ultimately myofibers (8-10). The level of eukaryotic initiation factor 2 (eIF2)associated glycoprotein p67 increases gradually when $\mathrm{C} 2 \mathrm{C} 12$ myoblasts differentiate into myotubes and peaks at the time of myoblasts fusion into myotubes (11). During this time period, p67 dissociates from eIF2 and associates with ERK1/2 leading to the increased phosphorylation of eIF2 $\alpha$, thus suppression of rates of global protein synthesis (12) and decreased phosphorylation of ERK1/2 that resulted into the inhibition of cell proliferation (13-15). Both events are essential for keeping myotubes in cell cycle arrested state (14). 
Rat p67 is 480 amino acid long and this could be divided into two segments - one is p26, the Nterminal 1-107 amino acid long and the other is p52, which contains 108-480 amino acid segment. P52 segment folds to create a shallow groove, where conserved D251, D262, H331, E364, and E459 amino acid residues come together to create the substrate-binding pocket. Close to the substratebinding pocket, its $\mathrm{H} 231$ residue performs the intermolecular and intra-molecular proteolytic activity $(13,16)$. P26 segment of the molecule contains two lysine-rich domains I \& II, which are separated by an acidic residue-rich domain. These lysine-rich domains along with its acidic residue-rich domain are involved in the modulation of phosphorylation of several kinases and target proteins $(13,17)$. In addition to p67's roles in cell cycle arrest in the myotubes, it may be involved in growth and proliferation of myoblasts and during the time of fusion of myoblasts into myotubes. In myoblasts, ERK1/2 MAP kinases along with other signaling molecules provide growth factor mediated signal for proliferation $(3-4,8)$ and thus keeping them in cycling state. Growth factors also increase the expression and activity of lipid induced protein kinase C (cPKCalpha) in mammalian cells $(7,10)$. The activity of cPKCalpha gradually decreases as myogenic differentiation advances and maximum inhibition are essential during the fusion of myoblasts into multinucleated myotubes $(7,10)$.

To examine whether there is any relationship between the gradual increase of p67 level and gradual decrease of cPKCalpha expression and activity during differentiation of $\mathrm{C} 2 \mathrm{C} 12$ myoblasts into myotubes, we measured the level of cPKCalpha in $\mathrm{C} 2 \mathrm{C} 12$ myoblasts and myotubes constitutively expressing rat p67 and some of its mutants. We noticed that when compared with control cells cPKCalpha level is $~ 10$-fold higher in p67-expressing myoblasts and p67's lysine residue-rich domain I \& II and in some extend its N-terminal acidic residue-rich domain are essential for this increased cPKCalpha level. In addition, alanine substitution of p67's conserved D251 residue compensates the inhibitory effects of cPKCalpha expression due to mutation at the acidic residue-rich sequences. In differentiated $\mathrm{C} 2 \mathrm{C} 12$ myoblasts however, cPKCalpha level goes down $\sim 50 \%$ in p67-expressing as compared to control differentiated cells. In addition, the conserved D251 residue and lysine-rich domains I \& II and not the acidic residue-rich domain of p67 are involved in its activity to inhibit the cPKCalpha level in $\mathrm{C} 2 \mathrm{C} 12$ myotubes. Although, mutation at p67's acidic residue-rich domain showed the same effect on suppression of cPKCalpha level as the wild type rat p67, a second site mutant at $\mathrm{H} 331$ residue $(\mathrm{H} 331 \mathrm{~A})$ on this acidic domain increased cPKCalpha significantly, indicating that $\mathrm{H} 331$ residue may have positive effect on suppression of cPKCalpha level in $\mathrm{C} 2 \mathrm{C} 12$ myotubes. Altogether, our data suggest that gradual increase of p67 level in $\mathrm{C} 2 \mathrm{C} 12$ myoblasts is involved in the gradual decrease of cPKCalpha level and its activity during the differentiation of $\mathrm{C} 2 \mathrm{C} 12$ myoblasts into multinucleated myotubes.

\section{MATerials AND MethodS}

All chemicals used in this study were obtained from Sigma Chemicals (St. Louis, MO), Merck (Darmstadt, Germany), ICN Biomedicals, Inc. (Aurora, Ohio), Fisher Chemicals (New Jersey), or GIBCO-BRL (Rockville, MD). All enzymes used in this study were purchased from New England Biolabs (Beverly, MA). Molecular mass markers were purchased from BioRad.

\subsection{Antibodies}

A monoclonal antibody specific to cPKCalpha (Sc-80) was purchased from Santa Cruz Biotechnology. A monoclonal antibody specific to aActin was purchased from Sigma (Sigma Biochemicals, St. Louis, MO). Tagged secondary antibodies for Western blots were obtained with the ECL kit (Perkin Elmer).

\subsection{Site-Directed Mutagenesis and Subcloning into Mammalian Expression Vector}

A $~ 1.4 \mathrm{~kb}$ cDNA insert encoding the entire p67 coding region was produced by PCR using appropriate forward and reverse primers (5' TCCCCCGGGTGATGGCGGGCGTGGAAGAG 3' and 5' TCCCCCGGGAAGTTTTAATAGTCATCTCCTC 3' respectively) with the pGEM-p67 template (18). The resulting DNA fragment was gel purified, digested with SmaI, and ligated in M13mp18 (Stratagene) at the SmaI site. A single-stranded uracil template was made, and annealing reactions were performed with mutant oligonucleotides. A detailed description for the generation of specific p67 mutants and their sequence variations as compared to wild type rat p67 was reported (19). The cDNA inserts for p67 with mutations at specific domains or sites were isolated from the RF form of M13mp18, digested with XmaI, and the DNA inserts were ligated at the XmaI site of pEGFP-C3 vector (Clontech, Palo Alto, CA). Plasmids with sense orientation were selected for further analysis. The specific fusion junction between mutant cDNA of p67 and enhanced green fluorescent protein 

Myotubes

(EGFP) was confirmed by DNA sequencing. All procedures for manipulation of recombinant DNA were either published earlier (19) or followed from molecular cloning, a laboratory manual (20), and current protocols in molecular biology (21).

\subsection{Cell Culture and Generation of Stable Cell Lines}

$\mathrm{C} 2 \mathrm{C} 12$ mouse myoblasts (ATCC) cultures were maintained in growth medium as described (11-12, $14,22)$. 50-60\% confluent cultures of $\mathrm{C} 2 \mathrm{C} 12$ cells were transfected with plasmids complexed with Superfect following protocols described by the manufacturer (Qiagen). Cells constitutively expressing either EGFP or EGFP-fusions of p67 or its specific mutants were selected by treating cells with G418 following the procedures as described $(11-12,14,22)$. C2C12 myoblasts constitutively expressing EGFP, or its various in-frame fusion of rat p67 and its specific mutants were grown in growth medium to confluence and then allowed to differentiate into myotubes for $96 \mathrm{~h}$ in differentiation medium following the procedures as described $(11-12,14,22)$.

\subsection{Cell Lysate Preparation and Western Blot Analysis}

Procedures for preparation of cell lysates either from myoblasts or myotubes and Western blots were essentially the same as described $(11-12,14,22)$. Protein bands in Western blots were scanned and their intensities were quantitatively measured by NIH Image 162 software program.

\section{RESUlTS AND DisCUSSION}

We have examined the effects of constitutive expression of rat p67 and some of its mutants on expression of cPKCalpha in $\mathrm{C} 2 \mathrm{C} 12$ myoblasts. For this purpose, we analyzed the total proteins from stable cell lines constitutively expressing EGFP and EGFP-fusions of rat p67 and its mutants, D251A, D6/2, K1K2, D6/2+D251, and D6/2+H331A in a Western blot using a monoclonal antibody specific to cPKCalpha (Fig. 1). When compared with control cells expressing EGFP alone (Fig. 1A, lane 1), we found $\sim 10$-fold increase of cPKCalpha level in both p67-expressing and D251A-expressing myoblasts (Fig. 1A, lanes $2 \& 3$ ), near unchanged in D6/2-expressing cells (lane 4), 2-fold increase in K1K2-expressing cells (lane 5), $\sim 6$-fold increase in double mutant, D6/2+D251A-expressing cells (lane 6), and $~ 50 \%$ increase in another double mutant, D6/2+H331A-expressing cells (lane 7). These results indicate that p67 is involved in induced expression of cPKCalpha; Its N-terminal acidic residue-rich sequences and the lysine-rich domains I \& II have very strong effects on this induction. Since the D251A mutant and not H331A of p67 can rescue the dramatic effect(s) of D6/2 mutant, the D251 residue is also involved in the regulation of p67's induction of cPKCalpha expression.

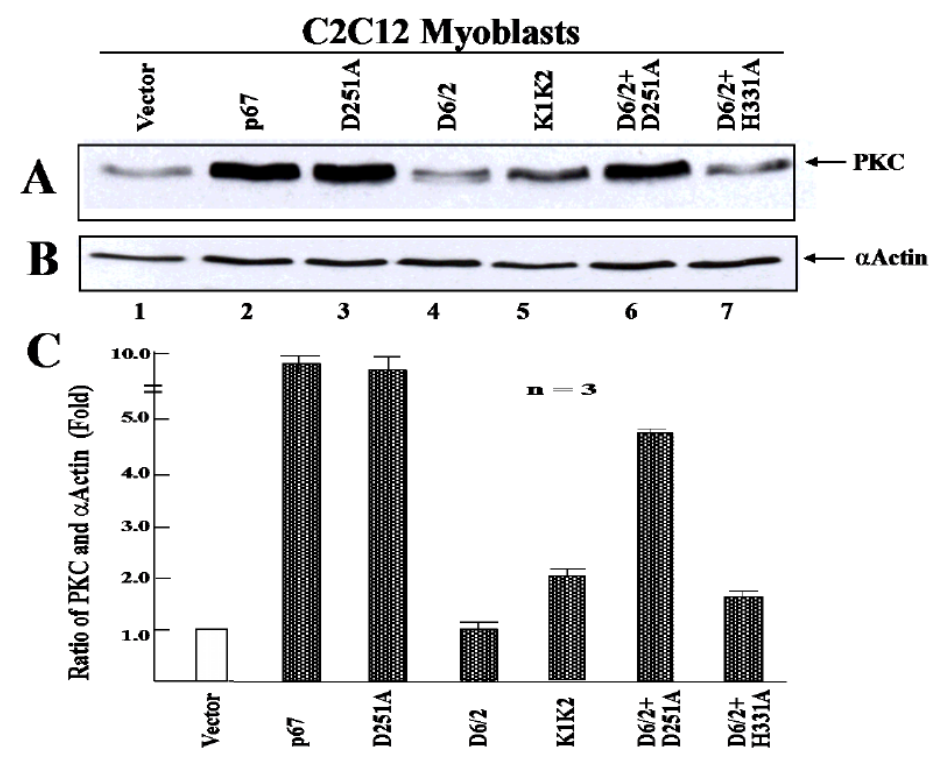

Fig1. Levels of cPKCalpha in C2C12 myoblasts constitutively expressing p67 and its mutants D251A, D6/2, $K 1 K 2, D 6 / 2+D 251 A$, and D6/2+H331A. Stable cell lines constitutively expressing EGFP expresion vector, and its in-frame fusions of rat $p 67$ or its various mutants were generated. Cell lysates from these cell lines were analyzed for the levels of cPKCalpha $(A)$ and $\alpha A c t i n(B)$ on Western blots using antibodies specific to these proteins. After scanning the band intensities from panel $A$ and panel $B$, the ratios of PKC and aActin were calculated and plotted on a graph $(C)$. The results are from three independent experiments $(n=3)$. 
Next, we examined the levels of cPKCalpha in $\mathrm{C} 2 \mathrm{C} 12$ differentiated cells constitutively expressing rat p67 and some of its mutants as mentioned above. For this purpose, the above-mentioned $\mathrm{C} 2 \mathrm{C} 12$ cell lines were allowed to differentiate in differentiation medium for $96 \mathrm{~h}$ and total proteins from these differentiated cells were analyzed for the level of cPKCalpha on a Western blot (Fig. 2). When compared with control differentiated cells we found that cPKCalpha level was decreased $\sim 50 \%$ both in p67- and D6/2-expressing differentiated cells (Fig. 2A, lane 2), 4.5-fold higher in D251Aexpressing differentiated cells (Fig. 2A, lane 3), 3.5-fold higher in both K1K2- and double mutant, D6/2+H331A- expressing differentiated cells (Fig. 2A, lanes 5 \& 7), and the other double mutant, D6/2+D251A-expressing differentiated cells showed $\sim 75 \%$ decrease in cPKCalpha level (Fig. 2A, lane 6). These results indicate that p67 is involved in the reduction of cPKCalpha level in the $\mathrm{C} 2 \mathrm{C} 12$ differentiated cells and its conserved D251 and H331 residues along with its N-terminal lysine-rich domains I \& II play important roles in the regulation of such p67's activity. In addition, the conserved D251 residue did not compensate the effect of lowering the cPKCalpha level by a p67 block mutant at the acidic residue-rich sequences.

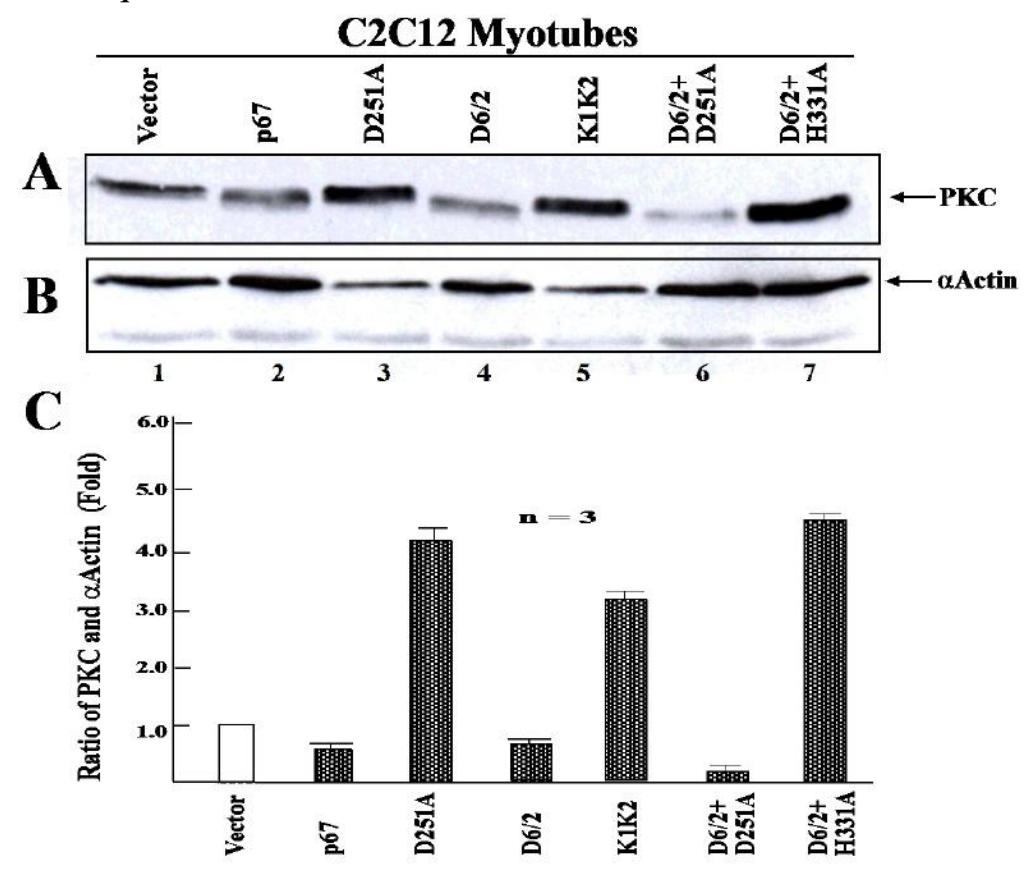

Fig2. Levels of cPKCalpha in C2C12 myotubes constitutively expressing p67 and its mutants D251A, D6/2, $K 1 K 2, D 6 / 2+D 251 A$, and D6/2+H331A. C2C12 myoblasts constitutively expressing EGFP vector or EGFPfusions of p67, D251A, D6/2, K1K2, D6/2+D251A, and D6/2+H331A mutants were allowed to differentiate into mytubes in differentiation medium for 96 h. Lysates were prepared after harvesting myotubes and analyzed for the levels of PKC (A) and $\alpha$ Actin (B) on Western blots using antibodies specific to these proteins. After scanning the band intensities from panel $A$ and panel B, the ratios of PKC and $\alpha A c t i n$ were calculated and plotted on a graph $(C)$. The results are from three independent experiments $(n=3)$.

cPKCalpha has very strong positive effects in cell growth and negative effects in cell differentiation $(7,23)$. In response to growth factors, like ERK1/2 MAP kinases cPKCalpha is activated and sends the signals to downstream effectors for cell proliferation (23). The level of p67 gradually increases in $\mathrm{C} 2 \mathrm{C} 12$ myoblasts differentiating into myotubes $(11-12,14)$. This increased level progressively binds to ERK1/2 kinases and inhibits their activation and activity significantly during the time of fusions of myoblasts into myotubes (11). During the same time of differentiation of $\mathrm{C} 2 \mathrm{C} 12$ myoblasts, p67 dissociates from eIF $2 \alpha$, increases eIF2 $\alpha$ phosphorylation to downregulate the global protein synthesis in the myotubes (12). In this study, we found almost 10-fold increase in cPKCalpha level in p67expressing $\mathrm{C} 2 \mathrm{C} 12$ myoblasts (Fig. 1) and $~ 50 \%$ lower in $\mathrm{C} 2 \mathrm{C} 12$ differentiated cells (Fig. 2) as compared to their basal levels in control cells. The N-terminal lysine residue-rich domains I \& II and in some extend acidic residue-rich sequences along with the conserved D251 residue but not H331 residue of p67 are involved in this upregulated expression of cPKCalpha in C2C12 myoblasts. Likewise, p67's N-terminal lysine residue-rich domains I \& II but not acidic residue-rich sequences along with both conserved D251 and H331 residues are involved in this downregulated expression of cPKCalpha in $\mathrm{C} 2 \mathrm{C} 12$ myotubes. These results thus indicate that p67 plays important role(s) in 
keeping $\mathrm{C} 2 \mathrm{C} 12$ myoblasts in cycling state and it is also involved in keeping $\mathrm{C} 2 \mathrm{C} 12$ myoblasts in differentiated state by inhibiting the expression of an inhibitor of differentiation such as cPKCalpha. In addition, p67 also modulates the expression and activity of MARCKS (24), one of the major substrates of cPKCalpha in a way to keep myoblasts in cycling state and myotubes in motile state. We however do not know whether these effects of p67 on the expression of cPKCalpha are direct or mediated by other protein(s).

P67's N-terminal lysine-rich domains I \& II and acidic residue-rich domain form two salt-bridge interactions (25-26). In absence of acidic domain, the lysine-rich domain I binds to the shallow groove created by p52 segment of p67 and the conserved acidic residues like D251, D262, E364, and E459 stabilize this binding. This brings the N-terminal sequence of p67 close to its catalytic H231 residue and gets proteolytically cleaved to generate p52 segment of p67 $(13,16)$. This p52 segment has autoproteolytic activity (16) indicating its involvement in intermolecular and intra-molecular proteolysis reactions. Since both lysine-rich domains and the conserved D251 residue are essential for p67's involvement in induced expression of cPKCalpha in C2C12 myoblasts and reduced expression of in $\mathrm{C} 2 \mathrm{C} 12$ myotubes, it is more likely that two different target proteins are involved in p67's functions in the regulation of cPKCalpha level in $\mathrm{C} 2 \mathrm{C} 12$ myoblasts and myotubes. One is the inhibitor of cPKCalpha expression that is possibly degraded by p52 segment in myoblasts and the other is the activator of cPKCalpha expression that is also degraded by p 2 segment in the myotubes.

\section{REFERENCES}

[1] Buckingham, M., and Rigby, P. W. J. (2014) Gene regulatory networks and transcriptional mechanisms that control myogenesis. Dev. Biol. 28, 225-238.

[2] Meloche, S., and Pouyssegur, J. (2007) The ERK1/2 mitogen-activated protein kinase pathway as a master regulator of the G1- to S-phase transition. Oncogene 26, 3227-3239.

[3] Gwang, T., Park, K., Kim, E., Son, C., Park, J., Nikawa, T., and Choi, I. (2013) Inhibition of C2C12 myotube atrophy by a novel HSP70 inducer, celastrol, via activation of Akt1 and ERK1/2 pathways. Arch. Biochem. Biophys. 537, 21-30.

[4] Raman, M., Chen, W., and Cobb, M. H. (2007) Differential regulation and properties of MAPKs. Oncogene 26, 3100-3112.

[5] Han, J. W., Lee, H.-J., Bae, G.-U., and Kang, J.-S. (2011) Promyogenic function of integrin/FAK signaling is mediated by Cdo, Cdc2, and MyoD. Cellular Signalling 23, 1162-1169.

[6] Goel, H. L., and Dey, C. S. (2002) cPKCalpha -regulated myogenesis is associated with increased tyrosine phosphorylation of FAK, Cas, and paxillin, formation of Cas-CRK complex, and JNK activation. Differentiation 70, 257-271.

[7] Schmitz-Peiffer, C. (2013) The tail wagging the dog - regulation of lipid metabolism by protein kinase C. FEBS J. 280, 5371-5383.

[8] Czifra, G., Szollosi, A., Nagy, Z., Boros, M., Juhasz, I., Kiss, A., Erdodi, F., Szebo, T., Kivacs, I., Torok, M., Kovacs, L., Blumberg, P. M., and Biro, T. (2015) Protein kinase C $\delta$ promotes proliferation and induces malignant transformation in skeletal muscle. J. Cell. Mol. Med. 19, 396-407.

[9] Hennige, A. M., Martin, H., Jurgen, M., Harald, S., Sartorius, T., Hoene, M., Lehmann, R., Weigert, C., Peter, A., Bornemann, A., Kroeber, S., Pujol, A., Franckhauser, S., Bosch, F., Schick, F., Lammers, R., and Haring, H.-U. (2010) Enforced expression of protein kinase C in skeletal muscle causes physical inactivity, fatty liver, and insulin resistance in the brain. J. Cell. Mol. Med. 14, 903-913.

[10] Gaboardi, G. C., Ramazzotti, G., Bavelloni, A., Piazzi, M., Fiume, R., Billi, A. M., Matteucci, A., Faenza, I., and Cocco, L. (2010) A role for cPKCalpha $\varepsilon$ during $\mathrm{C} 2 \mathrm{C} 12$ myogenic differentiation. Cell. Signalling 22, 629-635.

[11] Datta, B., Datta, R., Majumdar, A., and Ghosh, A. (2005) The stability of eukaryotic initiation factor 2-associated glycoprotein, p67 increases during skeletal muscle differentiation and that inhibits the phosphorylation of extracellular signal-regulated kinases 1 and 2. Exp. Cell Res. 303, 174-182. 
[12] Datta, B. and Datta, R. (2014) Phosphorylation of eukaryotic initiation factor $2 \alpha$ during differentiation of mouse myoblasts into myotubes is mediated by an unknown kinase. Intl. J. Adv. Res. Chem. Sci. 1, 1-5.

[13] Datta, B. (2015) Diversified roles of p67/MetAP2 as a regulator of cell growth and differentiation, in tumor suppression, and in obesity. Review, Curr. Topics Biochem. Res. 16, 41-52.

[14] Datta, B., Majumdar, A., Datta, R., and Balusu, R. (2004) Treatment of cells with the angiogenic inhibitor fumagillin results in increased stability of eukaryotic initiation factor 2 -associated glycoprotein, p67 and that inhibits the phosphorylation of extracellular signal-regulated kinases. Biochemistry 43, 14821-14831.

[15] Majumdar, A., Ghosh, A., Datta, S., Prudner, B., and Datta, B. (2010) P67/MetAP2 suppresses K-RasV12 mediated transformation of NIH3T3 mouse fibroblasts in culture and in athymic mice. Biochemistry 49, 10146-10157.

[16] Datta, B., Ghosh, A., Majumdar, A., and Datta, R., (2007) Auto-proteolysis of rat p67 generates several peptide fragments: The N-terminal p26 fragment is required for the protection of eIF2 $\alpha$ phosphorylation. Biochemistry 46, 3465-3475.

[17] Datta, B. (2009) Roles of P67/MetAP2 as a tumor suppressor - a review. Biochim. Biophys. Acta 1796:281-292.

[18] Wu, S., Gupta, S., Chatterjee, N., Hileman, R.E., Kinzy, T.G., Denslow, N.D., Merrick, W.C., Chakrabarti, D., Osterman, J.C., and Gupta, N.K. (1993) Cloning and characterization of complementary DNA encoding the eukaryotic initiation factor 2-associated $67-\mathrm{kDa}$ protein $\left(\mathrm{p}^{67}\right)$ J. Biol. Chem. 268, 10796-10801.

[19] Ghosh, A., Tammali, R., Balusu, R., Datta, R., Chattopadhyay, A., Bhattacharya, M., and Datta, B. (2014) Oligomerization of the eukaryotic initiation factor 2-associated glycoprotein p67 requires N-terminal 1-107 amino acid residues. Intl. J. Appl. Biotech. Biochem._4, 25-44.

[20] Ausubel, F. M., Brent R., Kingston R. E., Moore D. D., Seidman J. G., Smith J. A., Struhl K. (1992) Current Protocols in Molecular Biology, Vol. 1, section 8.1.

[21] Sambrook, J., Fritsch, E. F., and Maniatis, T. (1989) Molecular Cloning: A Laboratory manual, $2^{\text {nd }}$ edn., Cold Spring Harbor Laboratory Press, Cold spring Harbor, NY.

[22] Datta, B. Min, W., Burma, S., and Lengyel, P. (1998) Increase in p202 expression during skeletal muscle differentiation: Inhibition of MyoD protein expression and activity by p202. Mol. Cell. Biol. 18, 1074-1083.

[23] Garg, R., Benedetti, L. G., Abera, M. B., Wang, H., Abba, M., Kazanietz, M. G. (2014) Protein kinase $\mathrm{C}$ and Cancer: what we know and what we do not. Oncogene 33, 5225-5237.

[24] Datta, B, and Datta, R. (2016) Differential expression of MARCKS in C2C12 myoblasts and myotubes constitutively expressing p67. Submitted.

[25] Datta, B., Datta, R., Ghosh, A., and Majumdar, A. (2006) The binding between p67 and eukaryotic initiation factor 2 plays important roles in the protection of eIF2 $\alpha$ from phosphorylation by kinases. Arch. Biochem. Biophys. 452, 138-148.

[26] Datta, B., Datta, R., Ghosh, A., and Majumdar, A. (2004) Eukaryotic initiation factor 2associated glycoprotein, p67, shows differential effects on the activity of certain kinases during serum-starved conditions. Arch. Biochem. Biophys. 427, 68-78.

\section{AuthORS' BIOGRAPHY}

\section{Samit Kumar Datta, MD}

Current address: Lenox Hill Hospital, Manhattan, NY

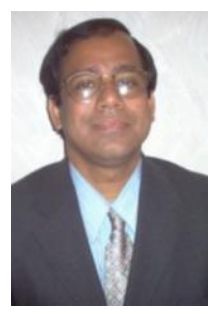

Bansidhar Datta, Ph. D.

Associate Professor, Department of Chemistry \& Biochemistry

Kent State University, Kent, OH 44240 